\title{
A novel potato microRNA stu-miR856 regulates mitogen-activated protein kinase genes contributing to drought tolerance
}

\author{
J.W. YANG ${ }^{1,2}$, X. ZHU ${ }^{2}$, S.G. $\mathrm{LI}^{2}$, X. TANG ${ }^{1,2}$, N. ZHANG ${ }^{1 *}$, and H.J. SI ${ }^{1,2}$ \\ College of Life Science and Technology, Gansu Agricultural University, Lanzhou 730070, P.R. China ${ }^{1}$ \\ Gansu Provincial Key Laboratory of Aridland Crop Science, Gansu Agricultural University, \\ Lanzhou 730070, P.R. China ${ }^{2}$
}

\begin{abstract}
Mitogen-activated protein kinases (MAPKs) are significant components of MAPK cascades, which play versatile roles in different transduction pathways to mediate stress adaptation. However, little information is known about post-transcriptional regulation of MAPK genes in plant under drought stress. MicroRNAs (miRNAs), a class of newly identified, short noncoding RNAs, regulate the expression of target genes in plant growth, development, and stress responses. In order to investigate the mechanism of miRNA regulating $M A P K$ genes in potato, we identified a novel potato miRNA with the sequence CGGCCTTAATAAGATGGTGAAG and named it as stu-miR856 depending on miRNA deep sequencing and bioinformatic analysis. Target prediction indicates that it can bind to the coding sequence region of two potato MAPK-like genes, and cleavage positions of them were also effectively validated by RNA ligase-mediated $5^{\prime}$ rapid amplification of cDNA ends assay. In addition, expressional analysis shows that stu-miR856 and its targets exhibited an opposite expression pattern: stu-miR856 expression significantly decreased while its target genes greatly increased in the different stages of drought treatment. The results indicate that a decreased expression of stu-miR856 might drive overexpression of two StMAPK genes family members, which may contribute to regulation of the drought adaptation of potato plants.
\end{abstract}

Additional key words: RLM-5' RACE, Solanum tuberosum, StMAPK.

\section{Introduction}

Plants often suffer by drought stress during their lifecycle (Nakashima et al. 2009), which restricts their growth and development. To overcome this challenge, crops have evolved defense systems to perceive environmental signals and respond to diverse environmental stresses. The mitogen-activated protein kinase (MAPK) cascade is one of the major signal transduction pathways (Ichimura et al. 2002), which phosphorylate various signaling molecules and transcription factors to regulate the expression of downstream genes (Droillard et al. 2004). The previous reports have indicated that $M A P K$ genes play an important role in response to various biotic and abiotic stresses (Ichimura et al. 2000, Jonak et al. 2002, Samuel et al. 2002, Xiong et al. 2003, Beckers et al. 2009, Gu et al. 2010). For example, 20 MAPKs have been identified from the Arabidopsis genome (Ichimura et al. 2002). Among these
AtMAPKs, three (AtMAPK3, AtMAPK4, and AtMAPK6) have been commendably studied, and AtMAPK4 responds to osmotic stress (Beckers et al. 2009), AtMAPK3 and AtMAPK6 belong to group A of Arabidopsis MAPK genes, which are intensely induced by abiotic stresses (Ichimura et al. 2000). The similar repertoires of $M A P K$ genes has also been found in other plants. For example in rice, $O S B W M K 1$ is one of the $M A P K$ gene families, and overexpression of $O S M A P K 5$ leads to cold and drought tolerance in transgenic plants (Xiong et al. 2003). In maize, a MAPK gene (ZmSIMK1) is strongly activated by drought and other stresses, and overexpression of ZmSIMK1 in transgenic plants results in overexpression of $R D 29 \mathrm{~A}$ and $P 5 C S 1$, which are stress-responsive marker genes $(\mathrm{Gu}$ et al. 2010). In tobacco, several $M A P K s$ have been verified to be activated under $\mathrm{NaCl}$ stress (Jonak et al. 2002). In tobacco, a $M A P K$ gene was induced by ozone, and it is important for effective mitigation of damage caused by

Submitted 4 October 2018, last revision 18 March 2019, accepted 8 April 2019.

Abbreviations: MAPK - mitogen-activated protein kinase; MFE - minimum folding free energy; MFEI - minimum folding free energy index; miRNA - microRNA; Mr - relative molecular mass; nr - non-redundant; RACE - rapid amplification of cDNA ends; PGSC potato genome sequencing consortium; pI - isoelectric point; qPCR - quantitative PCR; RLM - RNA ligase-mediated; UTR - untranslated region.

Acknowledgments: This research was supported by the National Natural Science Foundation of China (31460370, 31660416, and 31860400), the Discipline Construction Fund Project of Gansu Agricultural University (GSAU-XKJS-2018-172), and the Gansu Provincial Key Laboratory of Aridland Crop Science of Gansu Agricultural University (GSCS-2017-6).

* Corresponding author; fax: (+86) 931 7631875, e-mail: ningzh@gsau.edu.cn 
reactive oxygen species (Samuel et al. 2002). However, the post-transcriptional regulation of plant $M A P K$ genes is still not sufficiently known.

MicroRNAs (miRNAs) are 20 - 24 nucleotides long, single-stranded, and play a fundamental role in regulation of plant growth and developmental processes (JonesRhoades et al. 2006), as well as in adaptation to biotic and abiotic stresses (Sunkar et al. 2012, Kumar et al. 2018) by negatively regulating expression of target genes at the posttranscriptional level (Voinnet 2009), by degrading targeted gene mRNAs (Llave et al. 2002, Kidner and Martienssen 2005), and/or attenuate their translation (Chiou 2007). Since plant miRNAs were primarily identified in Arabidopsis (Llave et al. 2002, Park et al. 2002, Rhoades et al. 2002), so far, a large amount of plant miRNAs have been found, and an increasing number of miRNA target genes encoding transcription factors, protein kinase, important enzymes, and structural proteins have been confirmed (Jones-Rhoades et al. 2006). They are important in flower development, leaf morphogenesis, root and shoot development, auxin responses, signal transduction, and developmental phase change (Palatnik et al. 2003, Chapman et al. 2004, Mallory et al. 2004, Guo et al. 2005, Lauter et al. 2005, Wang et al. 2005). In addition, miR395, miR398, and miR399 also have been reported to be involved in responses to abiotic stresses (Sunkar and Zhu 2004, Jones-Rhoades et al. 2006, Sanan-Mishra et al. 2009). However, many experiments have been focused on model plants such as Arabidopsis thaliana and rice (Bartel and Bartel 2003, Bonnet et al. 2004, Griffiths et al. 2006). The knowledge of miRNA roles in responses to abiotic stresses in potato is still limited (Hwang et al. 2011a,b). Moreover, especially information about miRNA regulation mechanism of $M A P K$ genes in potato under drought stress is according to our knowledge totally unknown.

In order to find whether potato $M A P K$ genes are regulated by miRNAs under drought stress, miRNA deep sequencing and bioinformatics analysis were used in this study. A novel miRNA was identified in potato and named as stu-miR856. We propose that functional studies of stumiR856 can help us make a better understanding of the roles of miRNAs in regulation of potato response to drought and provide molecular evidence to improve the ability of drought resistance of potato.

\section{Materials and methods}

Bioinformatic analysis: All published plant miRNA sequences were downloaded from the public miRNA miRBase (http://microrna.sanger.ac.uk/) (miRBase Release 20.0, June, 2013)(Saini et al. 2008). Potato expressed sequence tags, genome survey sequences, and non-redundant sequences were retrieved from $N C B I$ (https://www.ncbi.nlm.nih.gov). The potato genome sequence was retrieved from the Potato genome sequencing consortium (PGSC; https://solanaceae.plantbiology.msu. edu/pgsc_download.shtml). The online software UNAFold (http://www.bioinfo.rpi.edu/applications/mfold/) was used to analyze the secondary structure and stability of RNAs.
The web-based software psRNATarget (http://plantgrn. noble.org/psRNA Target/) was used to find the target genes of miRNAs (Dai and Zhao 2011). The sequences of mRNA of known plant MAPKs were obtained from NCBI (http://www.ncbi.nlm.nih.gov/genbank). The softwares CluxtalX1.8 and MEGA5 were used to alignment analysis of amino acid sequences and construction of a phylogenetic tree.

Identification of stu-miR856 and its precursor in potato: Based on our previous miRNAs Solexa sequencing data in potato (Zhang et al. 2014) which were deposited in the sequence read archive of the NCBI database (accession No. SRP034924), the high-quality small RNA sequence (CGGCCTTAATAAGATGGTGAAG) was extracted from sequencing data and considered to be a novel potato miRNA. To ensure this sequence could be precisely accorded with a real miRNA, it was used as a query sequence to blast against the database of potato expressed sequence tags, genome survey sequence, and $\mathrm{nr}$; the parameters were defaulted. The closely matched sequences were used for predicting the secondary structure using the publicly available online software UNAFold (http:// www.bioinfo.rpi.edu/applications/mfold/old/rna/) with default parameters. In order to improve the authenticity of identified miRNAs and miRNA precursors, we chose sequences which had the folding structures with following parameter criteria: 1) the precursors of candidate miRNA can fold into a perfect or near-perfect hairpin structure; 2) the mature sequence of a candidate miRNA within one arm of the hairpin structure; 3) no loop or break in mature miRNA or miRNA* sequence of hairpin structures; 4) folding energy of predicted secondary structures must have a higher minimum folding free energy index (MFEI) and a more negative minimum folding free energy (MFE) than other types of RNAs, be greater than $60 \mathrm{bp}$ with MFE no greater than $-62.80 \mathrm{~kJ} \mathrm{~mol}^{-1}$; 5) The pre-miRNA sequence has a $\mathrm{G}+\mathrm{C}$ content of $50 \%$ or less (Zhang et al. 2006a,b, 2007). The sequences that agreed with these criteria were approved as candidate miRNA precursors for further analyses.

Cloning and identification of target sequences: We utilized psRNATarget to predict the target of stu-miR856 with default parameters. The result shows that two potato mRNA sequences (Table 1 Suppl.) were predicted as targets of stu-miR856 from the potato genome. To obtain the full-length sequences of the two genes, total RNA samples were isolated from leaves of potato (Solanum tuberosum L. cv. Phureja DM1-3) using a TRIzol reagent (Invitrogen, Carlsbad, USA). Quality and concentration of RNA samples were examined with a nanophotometer (IMPLEN, Munich, Germany). After treatment with DNase, first-strand cDNA synthesis was performed from the purified RNA samples following the manufacturer's protocol of a cDNA MMLV kit (Sangon, Shanghai, China). A double-stranded cDNA template was synthesized in a $20 \mathrm{~mm}^{3}$ reaction volume by DNA polymerase I following the first-strand cDNA sequence, which was used to amplify the full-length sequences of the two genes with the 
appropriate primers (Table 2 Suppl.). The PCR products were connected into a pGEM-T easy cloning vector and propagated in Escherichia coli DH5a (Promega, Madison, USA) for sequencing and conservation.

In order to identify the function of two cloned sequences, they were used as search queries to blast with the publicly available protein sequence (nr) database with $B L A S T X$ in NCBI (http://blast.ncbi.nlm.nih.gov/Blast. cgi? PROGRAM=blastx \& PAGE_TYPE=BlastSearch\& LINK LOC=blasthome). The BLASTP and TBLASTN (a basic local alignment search tool: http:// blast.ncbi. nlm.nih.gov) was available from $N C B I$ to use for further confirmation of genes, which were identified through BLAST searches with default parameters (Altschul et al. 1990). Protein sequences of identified genes were confirmed using the online programs Pfam database (protein family: http://pfam.Sanger. ac.uk/) with default parameters (Bateman et al. 2002, Letunic et al. 2012).

\section{Bioinformatic analysis of two StMAPK family members} in potato: Genomic sequences and chromosomal locations were obtained from the PGSC database (http://potatogenome.net/index.php/Main_Page). Gene structures (exons and introns) were investigated with splign (http://www.ncbi.nlm.nih.gov/sutils/splign) listed in NCBI (Kapustin et al. 2008). The isoelectric point (pI), molecular mass $(\mathrm{Mr})$, and other protein features including predicted amino acid sequences were calculated using the subprograms of Expasy (www.EXPASY.org). The multiple sequence alignments of protein sequences were carried out using the Clustal $X v 1.83$ program. A phylogenetic tree was constructed using MEGA version 5.0 (Tamura et al. 2011) with the neighbor-joining method. The dependability of distinct phylogenetic groups was assessed at 1000 replicates using bootstrap analysis and other default parameters.

Validation of miRNA-directed cleavage site using RNA ligase-mediated $5^{\prime}$ rapid amlification of cDNA ends PCR: In order to validate the putative truncated mRNAs of StMAPK10 and StMAPK11 at the stumiR856 cleavage sites, RNA ligase-mediated (RLM) $5^{\prime}$ rapid amplification of cDNA ends (RACE) assay was performed to experimentally validate predicted targets by using a GeneRaeer kit (Invitrogen) according to the manufacturer's instructions. Briefly, total RNA from potato leaves was obtained and ligated with a RNA adapter and reversely transcribed to yield cDNA.Nested RACEPCR was performed to amplify the 5'ends of StMAPK10 and StMAPK11 by PCR using the GeneRacer 5-adapter primer and the gene-specific primer (Table 3 Suppl.). The amplified PCR fragments were gel purified and cloned into a pMD18-T vector for sequencing to determine cleavage sites in the mRNAs of StMAPK10 and StMAPK11.

Plant growth and drought treatment: Potato (Solanum tuberosum L.) cv. Longshu 3 was planted in plastic pots, which were filled with a mixture of nursery soil and Vermiculite (1:1, v/v) in a greenhouse of Gansu Agricultural University under night/day temperatures of $22 / 26{ }^{\circ} \mathrm{C}$, a 16-h photoperiod, an irradiance of $200 \mu \mathrm{mol} \mathrm{m}{ }^{-2} \mathrm{~s}^{-1}$, and a relative humidity of $72 \%$. Forty pots of uniformly developed potato plants were grown in parallel. One month later, 18 pots, which plants showed uniform growth in, were chosen for continued cultivation. When the height of potato plants reached about $20 \mathrm{~cm}$, watering of a treated group was interrupted to achieve drought stress while control group was continually irrigated. Three replicate pots of each treatment were arranged in a completely randomized block design experiment. Fresh leaves were separately harvested from the treated and control groups every five days according to our previously used method (Yang et al. 2014) and immediately frozen and stored in $-80{ }^{\circ} \mathrm{C}$ for further RNA isolation.

Expressional analysis of stu-miR856 and targeted MAPK genes: For stu-miR856 expression analysis, total RNA was extracted from leaf samples of potato using a TRIzol reagent (Invitrogen). The cDNAs were synthesized using a One Step PrimeScript ${ }^{\circledR}$ miRNA cDNA synthesis kit (TaKaRa, Dalian, China) according to the manufacturer's instructions. Real-time quantitative PCR (qPCR) was carried out on an optical 96-well plate using a $S Y B R \AA$ Premix Ex TaqTM ${ }^{\mathrm{II}}$ kit (TaKaRa) with a 3000 Real-Time PCR system (Applied Biosystems, Foster City, USA). The PCR amplification was initiated with pre-denaturation at $95{ }^{\circ} \mathrm{C}$ for $10 \mathrm{~s}$ followed by 40 cycles of denaturation at $95^{\circ} \mathrm{C}$ for $10 \mathrm{~s}$, annealing at $60{ }^{\circ} \mathrm{C}$ for $30 \mathrm{~s}$, and extension at $72^{\circ} \mathrm{C}$ for $1 \mathrm{~min}$ (Feng et al. 2009). Each miRNA qPCR reaction was performed in a $20 \mathrm{~mm}^{3}$ reaction system containing $1 \mathrm{~mm}^{3}$ of a Uni-miR qPCR primer (provided in a $S Y B R{ }^{\circledR}$ Premix Ex TaqTM ${ }^{\mathrm{II}}$ kit), which was used as a forward primer, $1 \mathrm{~mm}^{3}$ of a stu-miR856 specific primer was used as a reverse primer (Table 2 Suppl.), $1 \mathrm{~mm}^{3}$ of cDNAs as a template, and $10 \mathrm{~mm}^{3}$ of SYBR ${ }^{\circledR}$ Premix Ex TaqTM ${ }^{\text {II }}$. The potato elongation factor 1-alpha gene was used as an internal reference gene to normalize the expression of stu-miR856. The reactions were performed with three replicates, and the control reactions were performed without reverse-transcribed cDNA.

For $M A P K$ genes expression analyses by real-time qPCR, a reaction was performed in a $20 \mathrm{~mm}^{3}$ PCR reaction system containing $10 \mathrm{~mm}^{3}$ of SYBR ${ }^{\mathrm{P} P \text { Pemix Ex TaqTM }}{ }^{\mathrm{II}}$ TM solution, $0.8 \mathrm{~mm}^{3}$ of each forward and reverse primers, and about $100 \mathrm{ng}$ of template cDNA. The components were mixed gently and incubated at $94{ }^{\circ} \mathrm{C}$ for 2 min followed by 40 cycles of $94{ }^{\circ} \mathrm{C}$ for $30 \mathrm{~s}, 60{ }^{\circ} \mathrm{C}$ for $34 \mathrm{~s}$, and $72{ }^{\circ} \mathrm{C}$ for $30 \mathrm{~s}$. All analyses were performed in three biological replicates with two technical replicates. The potato elongation factor 1-alpha gene was used as an internal control. The expression was calculated using the $2^{-\Delta \Delta} \mathrm{Ct}$ method, and standard deviations (SDs) were calculated from results of three independent experiments.

\section{Results}

Depending on miRNA high-throughput sequencing and bioinformatic analysis (Zhang et al. 2014), the small RNA sequence (CGGCCUUAAUAAGA UGGUGAAG) was 


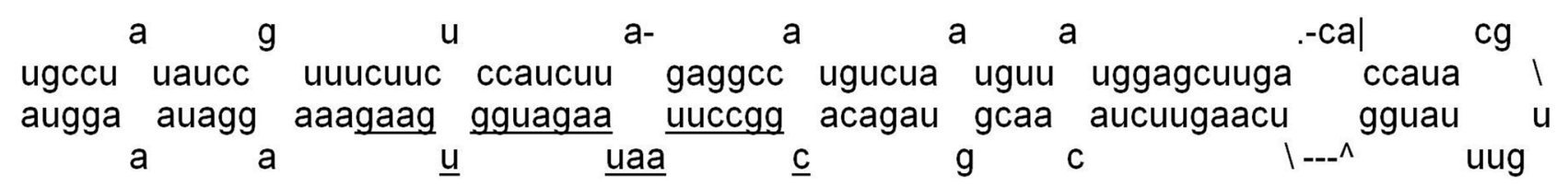

Fig. 1. Predicted hairpin secondary structures of stu-miR856. Mature microRNA (miRNA) sequences are underlined.

extracted from raw reads and considered to be a novel miRNA in potato. To ensure this sequence could be precisely accord with real miRNA, we performed BLAST strategy for the sequence and found that it was completely similar with the potato nucleotide collection (nr/nt) database sequence (GenBank accession No. AC232102.1). After predicting the secondary structure of candidate pre-miRNA sequence using the mFold software, the result shows that it can fold into a perfect hairpin structure of a typical miRNA family, a mature miRNA sequence within the 3' arm of the secondary structure, and had $5 \mathrm{nt}$ mismatches with the 5 ' arm sequence (the miRNA* sequence) of the hairpin structure (Fig. 1); no loop or break in the mature miRNA or miRNA* sequences in the predicted structure. The length of pre-miRNA was $215 \mathrm{nt}$. The miRNA precursor had a $\mathrm{G}+\mathrm{C}$ content of $33.5 \%$. The MFEI of secondary structure of predicted pre-miRNA was 1.13 and MFE was -81.2 . The detailed information is shown in Table 4 Suppl. Taking together, the result suggests that it was a real plant miRNA. It was named as stu-miR856.

Two potato mRNA sequences PGSC0003DMT400054750 and PGSC0003DMT400018188 (Table 1 Suppl.) have been predicted as the targets of stumiR856 from the potato genomics resource using the psRNATarget (http://plantgrn.noble.org/psRNATarget/). They were obtained from the potato genome database and were further aligned with the protein sequences $(n r)$ database to identify their function. A BLAST search and multi-alignment analysis with other well-studied plant (Arabidopsis and tomato) MAPKs were also performed. The Pfam tools were exploited to confirm putative $M A P K$ genes. The results reveal that those two sequences were highly related to well-studied plant $M A P K$ genes and had $M A P K$ domains (Fig. 1 Suppl.). The appropriate primers were designed and full-length mRNA sequences of these two MAPK-like genes were amplified (Table 2 Suppl.), They are highly homologous to known plant group D $M A P K$ family members. They were provisionally named as StMAPK10-like and StMAPK11-like based on the best homologous genes in Arabidopsis and tomato (Ichimura et al. 2002, Hamel et al. 2006). The full-length cDNA sequence of StMAPK10-like was 2107 bp with a 313 bp length of 5' UTR (untranslated region) and 12 bp length of 3' UTR, which contained a 1782 bp open reading frame encoding a protein of 593 amino acids. Its predicted Mr was $67.18 \mathrm{kDa}$, and $\mathrm{pI}$ was 9.22. The full-length cDNA sequence of StMAPK11-like was $2283 \mathrm{bp}$ with a 78 bp length of 5' UTR and $287 \mathrm{bp}$ length of 3' UTR. The cDNA sequence contained $1807 \mathrm{bp}$ length of open reading frame and encoded a protein of 603 amino acids. Predicted $\mathrm{Mr}$ was $68.23 \mathrm{kDa}$, and $\mathrm{pI}$ was 9.37. The detailed information is shown in Table 5 Suppl. The deduced peptide length, $\mathrm{pI}, \mathrm{Mr}$, and other protein features of these two StMAPK-likes were exceedingly close to well-studied $M A P K$ gene family members (Ichimura et al. 2002, Xiong and Yang 2003, Hamel et al. 2006, Gu et al. 2010).

To study the phylogenetic relationships of the two StMAPK-like genes and other reported plant $M A P K$ genes, the amino acid sequences of respective proteins of these two StMAPK-like genes, 16 SiMAPKs, and 19 AtMAPKs were performed using multiple alignment analysis by the $M E G A 5$ software. A phylogenetic tree was constructed (Fig. 2 Suppl.), and the results demonstrate that MAPKs are highly conserved in the plant kingdom. Based on the phylogenetic analysis of amino acid sequences and phosphorylation motifs, plant $M A P K S$ can be divided into four groups (A, B, C, and D) as monophyletic clades with at least $50 \%$ bootstrap support. The two potato StMAPKlike genes were followed into group D of MAPKS.

The chromosomal location analysis determined that the stMAPK10-like gene was located on chromosome 10, and the StMAPK11-like gene on chromosome 7 of potato. Gene structure prediction indicates that the StMAPK10-like and StMAPK11-like genes had 10 and 9 exons, respectively. The first exon of StMAPK10-like was 504 bp in length, whereas StMAPK11-like had a 718 bp first exon. Seven exons of two the StMAPK genes were highly conserved in length with the lengths of $410,140,60,150,156,212$, and 102 bp, respectively (Fig. 2), which were extremely close to well-studied plant group D of $M A P K$ family members (Nakashima et al. 2009). This conserved exon numbers in each subgroup among different species indicate their close evolutionary relationship and the introduced classification of subgroups.

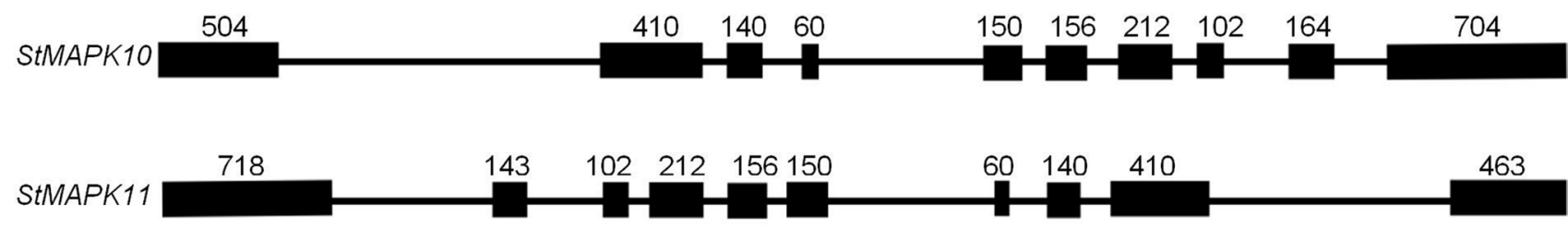

Fig. 2. Gene structures of StMAPK-like genes. Introns and exons a represented by lines and filled boxes, respectively. The numbers above the exons indicate the length $[\mathrm{bp}]$ of the exons. 
Fig. 3. The RNA ligase-mediated 5' rapid amplification of cDNA ends (RACE) verification of target mRNA cleavage sites generated by stu-miR856. Fractions within parentheses mean the proportions of 5' RACE clones showing these cleavage sites out of all sequenced clones.

The two potato MAPK genes (StMAPK10 and StMAPK11) were predicted as putative targets of stumiR856 by aligning the sequence of stu-miR856 to the potato transcript sequences. To validate the interaction

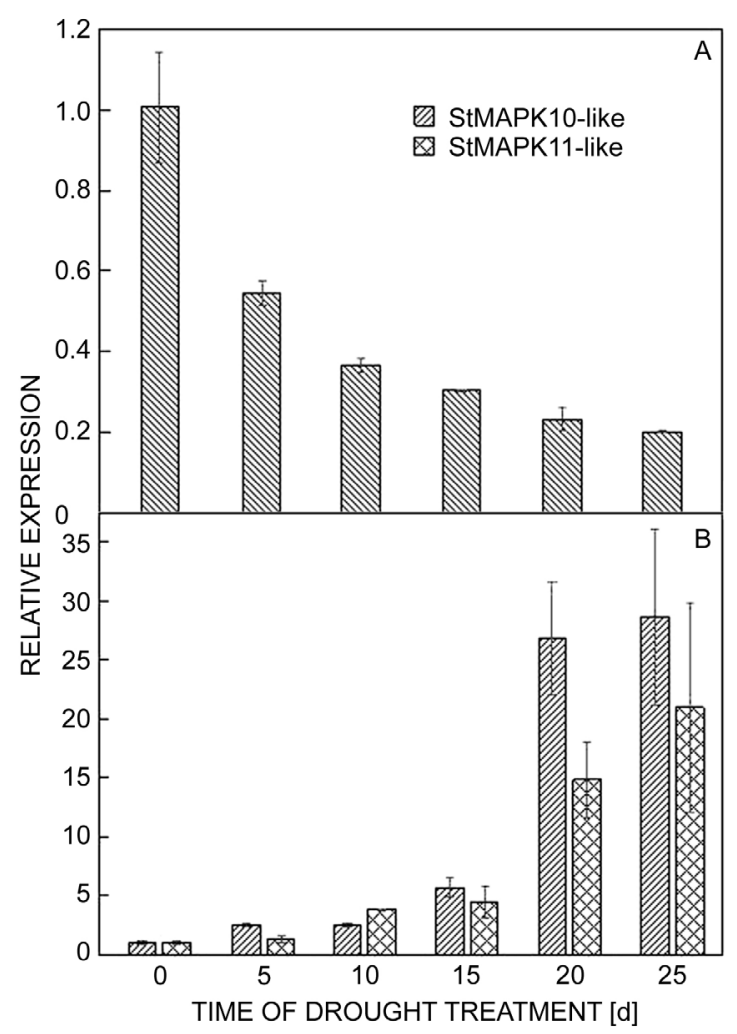

Fig. 4. Expression patterns of stu-miR856 $(A)$ and potential target genes $(B)$. Relative expressions of stu-miR856 and their potential target genes (StMAPK10-like and StMAPK11-like) were measured by real-time quantitative PCR in six periods of drought treatment. The expression of each gene in $0 \mathrm{~d}$ was set to 1 , and following expressions were quantified relative to it using the $2^{-\Delta \Delta \mathrm{Ct}}$ method. Elongation factor 1-alpha was used as an endogenous control. Means \pm SDs from three independent experiments. between stu-miR856 and the predicted targets (StMAPK10 and StMAPK11) in vivo, we performed RLM-5' RACE assay to amplify the cleavage positions of StMAPK10 and StMAPK11 transcripts, and found that most cleavage sites of the mRNA fragments were located at the $10^{\text {th }}$ nucleotide position of the stu-miR856 binding regions (Fig. 3), which suggests that StMAPK10 and StMAPK11 are targeted by stu-miR856 in potato.

To confirm the miRNAs obtained from computational prediction, real-time qPCR was used to validate the expression patterns of stu-miR856 and their respective target genes StMAPK10-like and StMAPK11-like. The qPCR detected the positive signal of stu-miR856, and it displayed an opposite expression pattern of StMAPK10like and StMAPK11-like during different periods od drought stress. The expression of stu-miR856 greatly decreased (Fig. 4A), while the expressions of its targets obviously increased (Fig. 4B). Therefore, stu-miR856 was considered to regulate overexpression of mitogenactivated protein kinase genes in response to drought stress in potato.

\section{Discussion}

Drought is one of the main environmental stresses that can severely affect the growth and development of crops and often causes reduction or total loss of their production. Plants adapt to drought stress by various biochemical and physiological changes and by an array of gene expression regulations. Over the past few years, a large number of drought-inducible genes have been identified in model plants (Kawasaki et al. 2001, Rabbani et al. 2003). Expression patterns of these genes contribute to elucidate the natural molecular mechanism of drought responses of plants (Kasuga et al. 2007). The MAPK is the terminal component of the MAPK signaling transduction cascades and plays an important role in integrating, amplifying, and channeling signals from both intracellular response and extracellular stimuli (Nadarajah and Sidek 2010). The 
important regulation of this signaling module can promote cloning and functional identification of $M A P K$ genes from potato and uncover the regulation mechanisms of the MAPK cascade under drought stress.

The miRNAs are a class of newly identified short noncoding RNAs, which accurately regulate the expression of target genes at the post-transcriptional level. Since the first miRNA was reported as a regulator of gene expression in Caenorhabditis elegans (Lee and Ambros 2001), to date, hundreds of miRNAs have been isolated in eukaryotic organisms. In plants, an increasing evidence indicates that miRNAs can regulate gene expressions by targeting mRNAs for cleavage or repressing translation to play important regulatory roles during plant development, growth, and stress responses.

A high-throughput sequencing technology has been reported to identify differentially expressed miRNAs at the whole transcriptome level. In the previous work using potato miRNA deep sequencing, we identified a novel potato miRNA stu-miR856 from raw reads (Zhang et al. 2014). Bioinformatics analysis showed that it is similar with the potato $n r / n t$ database sequence and can be folded to the typical hairpin structure of the miRNA family. The length of mature miRNAs was $22 \mathrm{nt}$, which is in accord with the typical length of mature miRNAs, and it had five mismatches with the opposite miRNA* sequence in the other arm, less than 6 nt mismatched (Griffiths et al. 2006). The length of a precursor was $215 \mathrm{nt}$, which is also in agreement with the normal length of pre-miRNA sequence, which had 60 to $400 \mathrm{nt}$ in length (Zhang et al. 2006a). The A+U content of the miRNA precursor was $33.5 \%$ (Table 4 Suppl.), which confirms the notion that miRNA precursor sequences have less $\mathrm{G}+\mathrm{C}$ content than $\mathrm{A}+\mathrm{U}$ content (Guddeti et al. 2005). Previous studies also showed that a low free energy is one of the important characteristics of typical secondary structure of pre-miRNAs (Seffens and Digby 1999). Although the secondary structure of predicted pre-miRNA has a lower folding free energy, MFE mainly depends on the length of pre-miRNAs (Bonnet et al. 2004). To better distinguish miRNAs from other types of RNAs using MFE and avoid the effect of the length of pre-miRNAs, a new criterion called MFEI was established (Altschul et al. 1990), and miRNA precursors should have a low folding free energy and a high MFEI (Zhang et al. 2006b). It is well known that the average MFEI of previously known plant miRNA precursors is 0.97 , and no other type of RNAs with higher than 0.85 MFEI have been found. This suggested that MFEI is useful criterion to distinguish miRNAs from other non-coding and coding RNAs. From the present prediction, the precursor of identified miRNA had MFEI of 1.13 , which is considerably more than 0.85 , and most likely, it had to be a potato miRNA (Zhang et al. 2006b). In order to make certain that the predicted miRNA really exist in potato, the predicted miRNA was verified and quantified for differently treated potato samples using Applied Biosystems SYBR ${ }^{\circledR}$ Premix Ex TaqTM ${ }^{\mathrm{II}}$ assay. The result shows that it was successfully detected and differentially expressed under drought stress.

The MAPK signal transduction module is the terminal component of MAPK signaling transduction cascades and plays an important role in diverse processes including development, defense, and abiotic stress responses (Mockaitis and Howell 2000, Munnik and Meijer 2001, Yuasa et al. 2001, Cardinale et al. 2002, Ren et al. 2002, Teige et al. 2004, Kumar et al. 2008, Zhang et al. 2012). The importance of this signaling module prompted cloning and functional identification of more $M A P K$ genes in plants. However, to date, a limited number of $M A P K$ genes have been identified and functionally characterized in Arabidopsis, rice, poplar, tobacco, and tomato (Asai et al. 2002, Rao et al. 2010, Kong et al. 2012, Zhang et al. 2013). Especially, only a few data of MAPK genes in potato are available. In the present study, we carried out sequence homology analysis to identify members of drought responsive $M A P K$ gene family members in potato and obtained two StMAPK-like genes from potato cv. Longshu-3. They belong to plant MAPKs of group D. Furthermore, the two members have been characterized on the basis of their chromosomal location, phylogenetic relationships, exon/intron organization, and drought stress-induced expressions. The evolutionary development of those two StMAPK-likes may follow the same pattern as $M A P K s$ of other plant species.

Previous researches have indicated that miRNAs play important roles in plant response to various environmental stresses and an increasing number of abiotic stress-induced miRNAs and their target genes have been identified. For example, the target gene of miR319 encodes myeloblastosis (MYB) transcription factor (Lu et al. 2005), which is widely involved in various stress responses. The miR319 is downregulated by cold stress in rice, and a decreased expression of rice miR319 can lead to the overexpression of MYB transcription factors. Overexpression of cold-induced rice MYB familly members (Os-MYB4 and $O s-M Y B 3 R-2)$ can help plant to enhance freezing tolerance (Vannini et al. 2004, Dai et al. 2007). Zhou et al. (2013) found that transgenic creeping bentgrass with overexpressed OsamiR319a shows morphological changes and an enhanced drought tolerance. The target gene of miR395 is ATP sulfurylase, which is a widespread enzyme that catalyzes sulfate activation in response to extracellular stress signal, and overexpression of ATP sulfurylase helps plant to increase glutathione content and stress tolerance (Noctor et al. 1998). The miR398 is down-regulated under drought stress in tomato (Luan et al. 2014), and the target gene of miR398 is copper/zinc superoxide dismutase, a scavenger of reactive oxygen species (Sunkar et al. 2006). Downregulation of miR398 results in an increase of copper/ zinc superoxide dismutase expression and tolerance to oxidative stress (Ding et al. 2013). All in all, the expression patterns of miRNAs and its targets are frequently used as an indicator for functional research of miRNAs.

In this study, the expression profiles of stu-miR856 and its predicted targets at different drought treatment periods were examined by real-time qPCR. The result shows that stu-miR856 had a negative expression pattern with its target genes. The stu-miR856 was down-regulated after experiencing a longer drought stress treatment, whereas the expressions of target genes were up-regulated. We suggest 
that the a lower expression of stu-miR856 may result in overexpression of StMAPK10-like and StMAPK11-like target genes, which can help plant response to drought stress. There are four points supporting our findings. Firstly, the potato novel miRNA (stu-miR856) had been identified and its expression significantly decreased under drought stress. Secondly, stu-miR856 had a binding site on the coding sequence of two identified StMAPK-like family members, and those two StMAPK-like genes have been identified and cloned from potato. Thirdly, cleavage sites of StMAPK10-like and StMAPK11-like were validated at the predicted stu-miR856 target sites by RLM-5'RACE assay (Fig. 3). Further, stu-miR856 was down-regulated, and the expressions of those two StMAPK-like genes were up-regulated under drought stress compared with the control, and they showed negative expression patterns, which is similar to the previously identified miRNAs and their target genes (Dai et al. 2007). Therefore, we suggest that stu-miR856 drove overexpression of StMAPK-like gene family members under drought stress in potato.

In conclusion, we identified stu-miR856 as a possible candidate miRNA which is down-regulated along with potato drought response. Overexpression of potato MAPKlike subfamily members, possible targets of stu-miR856, were also correlated with drought stress. These finding indicate that down-expression of stu-miR856 may drive overexpression of $M A P K$-like genes, which can help potato plant to adapt to drought stress.

\section{References}

Altschul, S.F., Gish, W., Miller, W., Myers, E.W., Lipman, D.J.: Basic local alignment search tool. - J. mol. Biol. 215: 403410, 1990.

Asai, T., Tena, G., Plotnikova, J.: MAP kinase signaling cascade in Arabidopsis innate immunity. - Nature 415: 977-983, 2002.

Bartel, B., Bartel, D.P.: MicroRNAs: at the root of plant development? - Plant Physiol. 132: 709-717, 2003.

Bateman, A., Birney, E., Cerruti, L.: The pfam protein families database. - Nucl. Acids Res. 30: 276-280, 2002.

Beckers, G.J., Jaskiewicz, M., Liu, Y.: Mitogen-activated protein kinases 3 and 6 are required for full priming of stress responses in Arabidopsis thaliana. - Plant Cell 21: 944-953, 2009.

Bonnet, E., Wuyts, J., Rouze, P., Van de Peer, Y.: Detection of 91 potential conserved plant microRNAs in Arabidopsis thaliana and Oryza sativa identifies important target genes. - Proc. nat. Acad. Sci. USA 101: 11511-11516, 2004.

Bonnet, E., Wuyts, J., Rouze, P., Van, P.Y.: Evidence that microRNA precursors, unlike other non-coding RNAs, have lower folding free energies than random sequences. Bioinformatics 20:2911-2917, 2004.

Cardinale, F., Meskiene, I., Ouaked, F., Hirt, H.: Convergence and divergence of stress-induced mitogen-activated protein kinase signaling pathways at the level of two distinct mitogenactivated protein kinase kinases. - Plant Cell 14: 703-711, 2002.

Chapman, E.J., Prokhnevsky, A.I., Gopinath, K., Dolja, V.V., Carrington, J.C.: Viral RNA silencing suppressors inhibit the microRNA pathway at an intermediate step. - Genes Develop. 18: 1179-1186, 2004.

Chiou, T.J.: The role of microRNAs in sensing nutrient stress. Plant Cell Environ. 30: 323-332, 2007.
Dai, X., Xu, Y., Ma, Q.: Overexpression of an $R 1 R 2 R 3 M Y B$ gene, $O S M Y B 3 R-2$, increases tolerance to freezing, drought, and salt stress in transgenic Arabidopsis. - Plant Physiol. 143: 1739-1751, 2007.

Dai, X., Zhao, P.X.: psRNATarget: a plant small RNA target analysis server. - Nucl. Acids Res. 39: W155-W159, 2011.

Ding, Y., Tao, Y., Zhu, C.: Emerging roles of microRNAs in the mediation of drought stress response in plants. - J. exp. Bot. 64: 3077-3086, 2013.

Droillard, M.J., Boudsocq, M., Barbier-Brygoo, H., Lauriere, C.: Involvement of MPK4 in osmotic stress response pathways in cell suspensions and plantlets of Arabidopsis thaliana: activation by hypoosmolarity and negative role in hyperosmolarity tolerance. - FEBS Lett. 574: 42-48, 2004.

Feng, J., Wang, K., Liu, X., Chen, S., Chen, J.: The quantification of tomato microRNAs response to viral infection by stemloop real-time RT-PCR. - Gene 437: 14-21, 2009.

Griffiths, J.S., Grocock, R.J., Van, D.S., Bateman, A., Enright, A.J.: miRBase: microRNA sequences, targets and gene nomenclature. - Nucl. Acids Res. 34: D140-D144, 2006.

Gu, L., Liu, Y., Zong, X., Liu, L., Li, D.P., Li, D.Q.: Overexpression of maize mitogen-activated protein kinase gene, ZmSIMK1 in Arabidopsis increases tolerance to salt stress. - Mol. Biol. Rep. 37: 4067-4073, 2010.

Guddeti, S., Zhang, D.C., Li, A.L., Leseberg, C.H., Kang, H., Li, X.G., Zhai, W.X., Johns, M.A., Mao, L.: Molecular evolution of the rice miR395 gene family. - Cell. Res. 15: 631-638, 2005.

Guo, H.S., Xie, Q., Fei, J.F., Chua, N.H.: MicroRNA directs mRNA cleavage of the transcription factor $N A C 1$ to down regulate auxin signals for Arabidopsis lateral root development. - Plant Cell 17: 1376-1386, 2005.

Hamel, L.P., Nicole, M.C., Sritubtim, S., other authors?et al.: Ancient signals: comparative genomics of plant MAPK and MAPKK gene families. - Trends Plant Sci. 11: 192-198, 2006.

Hwang, E.W., Shin, S.J., Park, S.C., Jeong, M.J., Kwon, H.B.: Identification of miR17 family members and their putative targets responding to drought stress in Solanum tuberosum. Genes Genom. 33: 105-110, 2011a.

Hwang, E.W., Shin, S.J., Yu, B.K., Byun, M.O., Kwon, H.B.: miR171 family members are involved in drought response in Solanum tuberosum. - J. Plant Biol. 54: 43-48, 2011 b.

Ichimura, K., Mizoguchi, T., Yoshida, R., Yuasa, T., Shinozaki, K.: Protein phosphorylation and dephosphorylation in environmental stress responses in plants. - Adv. Bot. Plant Pathol. 32: 355-377, 2000.

Ichimura, K., Mizoguchi, T., Yoshida, R., Yuasa, T., Shinozaki, $\mathrm{K}$. : Mitogen-activated protein kinase cascades in plants: a new nomenclature. - Trends Plant Sci. 7: 301-308, 2002.

Jonak, C., Ökrész, L., Bogre, L., Hirt, H.: Complexity, cross talk and integration of plant MAP kinase signalling. - Curr. Opin. Plant Biol. 5: 415-424, 2002.

Jones-Rhoades, M.W., Bartel, D.P., Bartel, B.: MicroRNAs and their regulatory roles in plants. - Annu. Rev. Plant Biol. 57: 19-53, 2006.

Kapustin, Y., Souvorov, A., Tatusova, T., Lipman, D.: Splign: algorithms for computing spliced alignments with identification of paralogs. - Biol. Direct. 3: 1-13, 2008.

Kasuga, M., Liu, Q., Miura, S., Yamaguchi, S.K., Shinozaki, K.: Improving plant drought, salt, and freezing tolerance by gene transfer of a single stress-inducible transcription factor. - Nat. Biotechnol. 17: 287-291, 2007.

Kawasaki, S., Borchert, C., Deyholos, M., Wang, H., Brazille, S.: Gene expression profiles during the initial phase of salt stress in rice. - Plant Cell 13: 889-905, 2001.

Kidner, C.A., Martienssen, R.A.: The developmental role of microRNA in plants. - Curr. Opin. Plant Biol. 8: 38-44, 2005. 
Kong, F., Wang, J., Cheng, L., Liu, S., Wu, J., Peng, Z., Lu, G.: Genome-wide analysis of the mitogen- activated protein kinase gene family in Solanum lycopersicum. - Gene 499: 108-120, 2012.

Kumar, K., Rao, K.P., Sharma, P., Sinha, A.K.: Differential regulation of rice mitogen activated protein kinase kinase (MKK) by abiotic stress. - Plant Physiol. Biochem. 46: 891897, 2008.

Kumar, A., Farooqi, M.S., Mishra, D.C., Kumar, S., Rai, A., Chaturvedi, K.K., Lal, S.B., Sharma, A.L.: Prediction of miRNA and identification of their relationship network related to late blight disease of potato. - Microrna 7: 11-19, 2018.

Lauter, N., Kampani, A., Carlson, S., Goebel, M., Moose, S.P.: MicroRNA172 down-regulates glossy15 to promote vegetative phase change in maize. - Proc. nat. Acad. Sci. USA. 102: 9412-9417, 2005.

Lee, R.C., Ambros, V.: An extensive class of small RNAs in Caenorhabditis elegans. - Science. 294: 862-864,2001.

Letunic, I., Doerks, T., Bork, P.: SMART 7: recent updates to the protein domain. - Nucl. Acids Res. 40: 302-305, 2012.

Llave, C., Kasschau, K.D., Rector, M.A., Carrington, J.C.: Endogenous and silencing-associated small RNAs in plants. - Plant Cell 14: 1605-1619, 2002.

Lu, Y.H., Sun, R., Shi, C., Clark, L., Li, V.L.: Novel and mechanical stress responsive microRNAs in Populus trichocarpa that are absent from Arabidopsis, - Plant Cell 17: 2186-2203, 2005.

Luan, Y., Wang, W., Liu, P.: Identification and functional analysis of novel and conserved microRNAs in tomato. - Mol. Biol. Rep. 41: 5385-5394, 2014.

Mallory, A.C., Dugas, D.V., Bartel, D.P., Bartel, B.: MicroRNA regulation of NAC domain targets is required for proper formation and separation of adjacent embryonic, vegetative, and floral organs. - Curr. Biol. 14: 1035-1046, 2004.

Mockaitis, K., Howell, S.H.: Auxin induces mitogenic activated protein kinase (MAPK) activation in roots of Arabidopsis seedlings. - Plant J. 24: 785-796, 2000.

Munnik, T., Meijer, H.J.: Osmotic stress activates distinct lipid and MAPK signaling pathways in plants. - FEBS Lett. 498: 172-178, 2001.

Nadarajah, K., Sidek, H.M.: The green MAPKs. - Asian J. Plant Sci. 9: 1-10, 2010.

Nakashima, K., Ito, Y., Yamaguchi-Shinozaki, K.: Transcriptional regulatory networks in response to abiotic stresses in Arabidopsis and grasses. - Plant Physiol. 149: 88-95, 2009.

Noctor, G., Arisi, A.C., Jouanin, L., Foye, C.H.: Manipulation of glutathione and amino acid biosynthesis in the chloroplast, Plant Physiol. 118: 471-482, 1998.

Palatnik, J.F., Allen, E., Wu, X.: Control of leaf morphogenesis by microRNAs. - Nature 425: 257-263, 2003.

Park, W., Li, J., Song, R., Messing, J., Chen, X.: CARPEL FACTORY, a Dicer homolog, and HEN1, a novel protein, act in microRNA metabolism in Arabidopsis thaliana. - Curr. Biol. 12: 1484-1495, 2002.

Rabbani, M.A., Maruyama, K., Abe, H., Khan, M.A., Katsura, K.: Monitoring expression profiles of rice genes under cold, drought, and high-salinity stresses and abscisic acid application using cDNA microarray and RNA gel-blot analyses. - Plant Physiol. 133: 1755-1767, 2003.

Rao, K.P., Richa, T., Kumar, K., Raghuram, B., Sinha, A.K.: In silico analysis reveals 75 members of mitogen-activated protein kinase kinase kinase gene family in rice. - DNA Res. 17: 139-153, 2010.

Ren, D., Yang, H., Zhang, S.: Cell death mediated by MAPK is associated with hydrogen peroxide production in Arabidopsis. - J. biol. Chem. 277: 559-565, 2002.

Rhoades, M.W., Reinhart, B.J., Lim, L.P., Burge, C.B., Bartel,
B., Bartel, D.P.: Prediction of plant microRNA targets. - Cell 110: 513-520, 2002.

Saini, S., Dongen, H.K., Enrigh, S.V.: miRBase: tools for microRNA genomics. - Nucl. Acids Res. 36: D154-D158, 2008.

Samuel, M.A., Ellis, B.E.: Double jeopardy: both over-expression and suppression of a redox-activated plant mitogen-activated protein kinase render tobacco plants ozone sensitive. - Plant Cell 14: 2059-2069, 2002.

Sanan-Mishra, N., Kumar, V., Sopory, S.K., Mukherjee, S.K.: Cloning and validation of novel miRNA from basmati rice indicates cross talk between abiotic and biotic stresses. - Mol. gen. Genom. 282: 462-473, 2009.

Seffens, W., Digby, D.: mRNAs have greater negative folding free energies than shuffled or codon choice randomized sequences. - Nucl. Acids Res. 27: 1578-1584, 1999.

Sunkar, R., Kapoor, A., Zhu, J.K.: Posttranscriptional induction of two $\mathrm{Cu} / \mathrm{Zn}$ superoxide dismutase genes in Arabidopsis is mediated by downregulation of miR398 and important for oxidative stress tolerance. - Plant Cell 18: 2051-2065, 2006.

Sunkar, R., Li, Y.F, Jagadeeswaran, G.: Functions of microRNAs in plant stress responses. - Trends Plant Sci. 17: 196-203, 2012.

Sunkar, R., Zhu, J.: Novel and stress regulated microRNAs and other small RNAs from Arabidopsis. - Plant Cell 16: 2001 2019, 2004.

Tamura, K., Peterson, D., Peterson, N., Stecher, G., Nei, M., Kumar, S.: MEGA5: molecular evolutionary genetics analysis using maximum likelihood, evolutionary distance, and maximum parsimony methods. - Mol. Biol. Evol. 28: 2731$2739,2011$.

Teige, M., Scheikl, E., Eulgem, T.: The $M K K 2$ pathway mediates cold and salt stress signaling in Arabidopsis. - Mol. Cells 15: 141-152, 2004.

Vannini, C., Locatelli, F., Bracale, M., Magnani, E., Marsoni, M., Osnato, M., Mattana, M., Baldoni, E., Coraggio, I.: Overexpression of the rice Osmyb4 gene increases chilling and freezing tolerance of Arabidopsis thaliana plants. - Plant J. 37: 115-127, 2004.

Voinnet, O.: Origin, biogenesis, and activity of plant microRNAs. - Cell 136: 669-687. 2009.

Wang, J.W., Wang. L.J., Mao, Y.B., Cai, W.J., Xue, H.W., Chen, X.Y.: Control of root cap formation by microRNA-targeted auxin response factors in Arabidopsis. - Plant Cell 17: 2204$2216,2005$.

Xiong, L., Yang, Y.: Disease resistance and abiotic stress tolerance in rice are inversely modulated by an abscisic acidinducible mitogen-activated protein kinase. - Plant Cell 15: 745-759, 2003.

Yang, J.W., Zhang, N., Mi, X.X.: Identification of miR159s and their target genes and expression analysis under drought stress in potato. - Comput. Biol. Chem. 14: 204-213, 2014.

Yuasa, T., Ichimura, K., Mizoguchi, T., Shinozaki, K.: Oxidative stress activates ATMPK6, an Arabidopsis homologue of MAP kinase. - Plant Cell Physiol. 42: 1012-1016, 2001.

Zhang, B.H., Pan, X.P., Anderson, T.A.: Identification of 188 conserved maize microRNAs and their targets. - FEBS Lett. 580: 3753-3762, 2006a.

Zhang, B.H., Pan, X.P., Cox, S.B., Cobb, G.P., Anderson, T.A.: Evidence that miRNAs are different from other RNAs. - Cell. mol. Life Sci. 63: 246-254, 2006 b.

Zhang, B.H., Wang, Q.L., Wang, K.B., Pan, X.P., Liu, F., Guo, T.L., Cobb, G.P., Anderson, T.A.: Identification of cotton microRNAs and their targets. - Gene 397: 26-37, 2007.

Zhang, L., Li, Y., Lu, W., Meng, F., Wu, C.A., Guo, X.: Cotton GhMKK5 affects disease resistance, induces HR-like cell 
death, and reduces the tolerance to salt and drought stress in transgenic Nicotiana benthamiana. - J. exp. Bot. 63: 39353951, 2012.

Zhang, N., Yang, J., Wang, Z., Wen, Y., Wang, J., He, W., Liu, B., Si, H., Wang D.: Identification of novel and conserved microRNAs related to drought stress in potato by deep sequencing. - PLoS ONE 9: e95489. 2014.
Zhang, X.T., Cheng., T.C, Wang., G.H., Yan, Y.F, Xia, Q.Y.: Cloning and evolutionary analysis of tobacco MAPK gene family. - Mol. Biol. Rep. 40: 1407-1415, 2013.

Zhou, M., Li, D., Li, Z., Hu, Q., Yang, C., Zhu, L.: Constitutive expression of a miR319 gene alters plant development and enhances salt and drought tolerance in transgenic creeping bentgrass. - Plant Physiol. 161: 1375-91, 2013. 